\title{
HIV-1 molecular epidemiology evidence and transmission patterns in the Middle East and North Africa
}

\author{
Ghina Mumtaz, ${ }^{1}$ Nahla Hilmi, ${ }^{2}$ Francisca Ayodeji Akala, ${ }^{2}$ Iris Semini, ${ }_{1}^{3}$ \\ Gabriele Riedner, ${ }^{4}$ David Wilson, ${ }^{5}$ Laith J Abu-Raddad ${ }^{1,6,7}$
}

${ }^{1}$ Infectious Disease

Epidemiology Group, Weill

Cornell Medical College Oatar, Cornell University, Oatar Foundation, Education City, Doha, Oatar

${ }^{2}$ Human Development Sector, Middle East and North Africa Region, World Bank, Washington, DC, USA

${ }^{3}$ Joint United Nations

Programme on HIV/AIDS

Regional Support Team, Middle

East and North Africa, Cairo, Egypt

${ }^{4}$ Regional Office of the Eastern Mediterranean, World Health Organization, Cairo, Egypt ${ }^{5}$ Global HIV/AIDS Program, World Bank, Washington, DC, USA

${ }^{6}$ Department of Public Health, Weill Cornell Medical College, Cornell University, New York,

New York, USA

${ }^{7}$ Vaccine and Infectious Disease Institute, Fred Hutchinson

Cancer Research Center,

Seattle, Washington, DC, USA

Correspondence to

Dr Laith J Abu-Raddad, Infectious Disease Epidemiology Group, Weill Cornell Medical College - Oatar, Oatar Foundation, Education City, P.O. Box 24144, Doha, Oatar; lja2002@qatar-med.cornell.edu

Accepted 24 August 2010 Published Online First 29 October 2010

\section{(2) UNLOCKED}

This paper is freely available online under the BMJ Journals unlocked scheme, see http://sti. bmi.com/site/about/unlocked. xhtml

\begin{abstract}
The distribution of HIV-1 subtypes in a population tracks the spread and evolution of the epidemic. This study is a systematic review of all available evidence on HIV-1 molecular epidemiology and subtype distribution in the Middle East and North Africa. Sources of data included Medline and various institutional documents and databases. In several countries, a diverse distribution of HIV-1 subtypes was observed principally reflecting travel-related exogenous exposures. A trend for a dominant HIV-1 subtype was observed in a few other settings and was often linked to HIV transmission within specific high-risk groups such as subtype $A$ and CRF35_AD among injecting drug users and subtype $C$ among commercial sex networks. Multiple exogenous introductions of HIV-1 variants seemed common to all countries, as observed from the high diversity in subtypes, or the high genetic divergence among any specific subtype even if predominant. In several countries though, epidemic-type clustering of specific subtypes suggests established or nascent HIV epidemics among classic core risk groups for HIV infection. HIV prevention efforts in MENA must be prioritized for these high-risk groups.
\end{abstract}

HIV-1 is characterised by a high genetic variability and includes three groups, with the main group responsible for the HIV pandemic (group $M$ ) being further divided into several subtypes $(\mathrm{A}-\mathrm{K})$ and a number of mosaic strains known as circulating recombinant forms (CRF). ${ }^{1}$ With the exception of sub-Saharan Africa, where the largest diversity of subtypes and CRF has been observed, HIV-1 subtypes show a specific geographical distribution on the global map. ${ }^{23}$ Subtype $\mathrm{A}$ is the predominant variant in central and eastern Africa and in countries of the former Soviet Union. Subtype B is predominant in western and central Europe, the Americas, Australia, and is commonly found in several countries of Southeast Asia. ${ }^{2}$ Subtype C is the main genetic form in southern Africa and India, the two countries where more than $80 \%$ of the global HIV cases occur. Finally, CRF are the most predominant forms in Southeast Asia (CRF01_AE) and in west and central Africa (CRF02_AG). ${ }^{2}$

Molecular epidemiology investigation can be a powerful tool in developing sensitive diagnostic tools, in the management of individual infections, and most importantly in tracking transmission patterns and the spread and evolution of the epidemic. ${ }^{4}$ Molecular epidemiology research in the Middle East and North Africa (MENA) region is at an early stage of development and has yet to contribute to informed HIV prevention policy and programming. A recent review on the epidemiology of HIV in MENA indicates that in this region where HIV spread in the general population is very limited, there are emerging epidemics in high-risk populations including injecting drug users (IDU), men who have sex with men (MSM), and to a lesser extent female sex workers (FSW) in a few countries. $^{5}$ Exogenous HIV exposures among nationals linked to travel abroad also appear to be the dominant observed HIV transmission pattern in a few MENA countries. ${ }^{5}$ However, there remain many gaps in the understanding of HIV transmission pathways in MENA.

The objective of this work was to review for the first time all evidence on HIV virus diversity and subtype distribution in MENA countries to complement our knowledge of HIV epidemiology and to understand transmission patterns.

\section{METHODS}

This work was part of a comprehensive systematic review of all available data about HIV in MENA, which was complemented by another detailed search focused specifically on published HIV molecular epidemiology literature in MENA. The countries included in our definition of MENA are: Afghanistan, Algeria, Bahrain, Djibouti, Egypt, Iran, Iraq, Jordan, Kuwait, Lebanon, Libya, Morocco, Oman, Pakistan, Palestine, Oatar, Saudi Arabia, Somalia, Sudan, Syria, Tunisia, United Arab Emirates and Yemen. Considering geographical proximity and similarity in the sociocultural context, data were occasionally included on Mauritania, Israel and Turkey.

The main source of data for this investigation was the MENA HIV/AIDS Epidemiology Synthesis Project whose mandate was to collect and synthesise all available data on HIV, sexually transmitted infections (STI) and sexual behaviour in MENA. ${ }^{6}$ Details of this project and of the search strategy used in this comprehensive systematic review were described in a previous publication. ${ }^{5}$ In brief, we undertook a literature search of Medline (PubMed) using a strategy with both free text and MeSH headings and with no time limitation. The main search criteria in relevance to the current work were (('HIV Seropositivity' OR 'HIV' OR 'HIV Infections') AND ('Middle East' OR 'Islam' OR 'Arabs' OR 'Arab World' OR 'Africa, Northern' OR 'Mauritania' OR 'Sudan' OR 'Somalia' OR 
'Djibouti' OR 'Pakistan')). We also searched for relevant data in country-level reports and databases, governmental and nongovernmental organisations studies and publications, international organisations' reports and databases, as well as other institutional reports related to HIV and STI in MENA. ${ }^{5}$

The above generic search was complemented by a specific search of the Medline database on all HIV-1 molecular data in MENA. The MeSH search criteria used were ('HIV-1/classification' OR 'HIV-1/genetics') AND ('Middle East' OR 'Islam' OR 'Iran' OR 'Arabs' OR 'Arab World' OR 'Africa, Northern' OR 'Mauritania' OR 'Sudan' OR 'Somalia' OR 'Djibouti' OR 'Pakistan')). All relevant studies identified in this search were already covered by the wide-umbrella search of the Synthesis Project above. However, this search was undertaken and respective citations reviewed for further reassurance that no relevant data were missed.

Finally, we searched the Los Alamos HIV sequence database, which gathers global HIV sequence data stratified by geographical location, but without any information on data source and study methodology. ${ }^{7}$ Data from all MENA countries were extracted from this online database and compared with results of published studies.

\section{RESULTS}

\section{Status of the evidence}

As part of the comprehensive Medline search that was undertaken for the Synthesis Project, a total of 1092 citations on HIV in MENA were retrieved as of 29 May 2010, out of which 49 citations were specific to the molecular epidemiology search. After excluding non-relevant studies, there remained 24 studies that examined the nature of HIV-1 subtypes and suggested transmission pathways in the population (table 1). HIV-1 subtype data were also extracted from the Los Alamos HIV database for all MENA countries and suggested trends similar to those observed in published studies (table 2).

All the reviewed studies used standard and comparable methodologies ${ }^{41}$ in their molecular analyses, consisting of the amplification of specific genomic regions (mostly env, gag and/or pol) using PCR, followed by sequencing of the PCR products and phylogenetic tree analysis. It appears unlikely that there is bias in comparing findings between studies and over time due to differences in the methodology of molecular analyses.

\section{Settings with variability of subtypes}

A number of countries in MENA display a very diverse distribution of HIV-1 subtypes. This is the case of Lebanon where a complex HIV-1 subtype distribution pattern and high levels of intrasubtype diversity were found representing the travel history of its nationals, particularly to Europe and the Americas where subtype B (44\%) is acquired, and to Africa and the United Arab Emirates where most of the non- $B$ subtypes, especially subtype A (40\%), are acquired. ${ }^{18}$ An analogous trend of high strain diversity including many CRF was observed in Saudi Arabia. ${ }^{24-26}$ However, the identification of a subcluster with a new CRF in this country (CRF43_02G) could indicate a local transmission network. ${ }^{24}$ HIV-1 subtypes in Yemen also displayed a complex distribution, which appeared to be a mix of

Table 1 Distribution of HIV-1 subtypes in MENA as extracted from various studies

\begin{tabular}{|c|c|c|c|c|c|}
\hline Country & Study & Sample size & Year* & Genes & Subtype (\%) \\
\hline Afghanistan & Sanders-Buell ${ }^{8}$ & 4 IDU & $2005-6$ & Pol, Env & CRF35_AD (100) \\
\hline Algeria & Bouzeghoub $^{9}$ & 134 Isolates & NAt & Rt, Prot, Env & $\begin{array}{l}\text { B (56), CRF02_AG (12.7), CRF06_cpx (4) } \\
\text { Inter-CRF recombinants (9.7) }\end{array}$ \\
\hline \multirow[t]{2}{*}{ Djibouti } & Maslin $^{10}$ & 34 Isolates & 2002 & Pol, Env & C (73), CRF02_AG (18), D (6), A (3) \\
\hline & Lasky $^{11}$ & 33 French military personnel in Djibouti & NA† & Env & $C(48), B(33), A(15), E(3)$ \\
\hline \multirow[t]{5}{*}{ Iran } & Sarrami-Farooshani $^{12}$ & 46 IDU and 15 haemophiliacs in Teheran & 2003 & Gag, Env & $\begin{array}{l}\text { IDU: A (100) } \\
\text { Haemophiliacs: B (100) }\end{array}$ \\
\hline & Naderi $^{13}$ & 12 IDU in Mashhad & 2005 & Gag, Env & $A(100)$ \\
\hline & Soheilli ${ }^{14}$ & 13 Isolates, mostly IDU & NA† & RT, Prot, Env & CRF35_AD (100) \\
\hline & Mousavi $^{15}$ & 39 Isolates, mostly IDU & $2005-6$ & Pol & CRF35_AD (100) \\
\hline & Hamkar $^{16}$ & 42 Isolates, $36 \%$ IDU & 2009 & Pol & $\begin{array}{l}\text { A/D recombinants (48), B (43), A (5), } \\
\text { CRF01_AE (5) }\end{array}$ \\
\hline Israel/Palestine & Gehring $^{17}$ & 92 Isolates, 45\% MSM & NAt & Env & $B(98), A(1), D(1)$ \\
\hline Lebanon & Pieniazek $^{18}$ & 25 Isolates & 1996 & Prot, Gag, Env & $A(44), B(40), C(4), D(4), G(4)$, \\
\hline Libya & de Oliveira ${ }^{19}$ & 44 Children & 1998 & Gag & CRF02_AG $(100)$ \\
\hline \multirow[t]{3}{*}{ Morocco } & Elharti $^{20}$ & 200 Isolates & NA† & NA† & $\mathrm{B}(93 . \overline{5}), \mathrm{A}(1 \%), \mathrm{F}(0.5 \%)$ \\
\hline & $\mathrm{Abid}^{21}$ & 14 Isolates & NAt & Env, Tat & $\mathrm{B}(78.6 \%), \mathrm{A}(21.4 \%)$ \\
\hline & Bakhouch $^{22}$ & 98 Isolates & $2004-7$ & Rt, Prot & $\begin{array}{l}\text { B (74.6\%), CRF02_AG (15.5), CRF01_AE } \\
(4.2), G(2.8), C(1.4), \text { F2 (1.4\%) }\end{array}$ \\
\hline Pakistan & $\mathrm{Khan}^{23}$ & 34 IDU in Karachi & 2006 & Gag, Env & $A(100)$ \\
\hline \multirow[t]{2}{*}{ Saudi Arabia } & $\begin{array}{l}\text { Yamaguchi }^{24} \\
\text { Badreddine }^{25}\end{array}$ & 56 Saudi nationals in Riyadh & $2003-4$ & Gag, Pol, Env & $\begin{array}{l}\text { C (39.3), B (17.8), Intersubtype } \\
\text { recombinants (16.1), CRF43_02G (10.7), } \\
\text { CRF25_cpx (7.1), D (3.6), A (1.8), } \\
\text { CRF02_AG (1.8) }\end{array}$ \\
\hline & Alzahrani $^{26}$ & 12 Isolates in Dammam & $2004-7$ & Gag, Env & $\mathrm{C}(58 \%), \mathrm{B}(17 \%), \mathrm{A}(8 \%), \mathrm{D}(8 \%), \mathrm{G}(8 \%)$ \\
\hline Sudan & Hierholzer $^{27}$ & 30 Blood donors & $1998-9$ & Pol, Env & $\begin{array}{l}D(50), C(30), \text { recombinants }(10), A(6.7) \\
B(3.3)\end{array}$ \\
\hline Tunisia & Ben Halima ${ }^{28}$ & 25 Isolates & 1999 & Env & B (95), CRF02_AG (5) \\
\hline Turkey & Yilmaz $^{29}$ & 27 Isolates & NAt & Env & $\begin{array}{l}\mathrm{B}(70.4), \mathrm{A}(1 \overline{4} .8), \mathrm{F} 1 \text { (7.4), C (3.7), } \\
\mathrm{D}(3.7)\end{array}$ \\
\hline Yemen & Saad $^{30}$ & 19 Isolates & $2000-2$ & Pol & $\begin{array}{l}\mathrm{B}(47.3), \mathrm{C}(31.6), \mathrm{D}(10.5), \mathrm{A}(5.3), \mathrm{URF} \\
(5.3)\end{array}$ \\
\hline
\end{tabular}


Table 2 Distribution of HIV-1 subtypes in MENA as extracted from the Los Alamos HIV database ${ }^{7}$

\begin{tabular}{|c|c|c|}
\hline Country & No of isolates & Subtype $(\%)^{*}$ \\
\hline Afghanistan & 4 & 35_AD (100) \\
\hline Algeria & 478 & $\begin{array}{l}\text { B (56.3), CRF02_AG (18.0), CRF06_cpx } \\
\text { (17.2), G (2.7), } 206(2.7), D(1.3) \text {, other } \\
(1.9)\end{array}$ \\
\hline Djibouti & 92 & $\begin{array}{l}\text { C (64.1), CRF02_AG (26.1), D (5.4), } \\
\text { A (3.3), CRF01_AE (1.1) }\end{array}$ \\
\hline Egypt & 24 & B (91.7), 0 (4.2), CRF01_AE (4.2) \\
\hline Iran & 277 & $\mathrm{~A}(65), \mathrm{B}(32.9), \mathrm{C}(2.2)$ \\
\hline Israel/Palestine & 301 & $\begin{array}{l}\text { C (85.4), B (8.6), CRF02_AG (1.7), } \\
\text { A (1.3), other (3.0) }\end{array}$ \\
\hline Kuwait & 2 & $C(100)$ \\
\hline Lebanon & 59 & $\begin{array}{l}\mathrm{A}(40.7), \mathrm{B}(35.6), \mathrm{D}(6.8), \mathrm{U}(5.1), \mathrm{C}(3.4), \\
\mathrm{G}(3.4), \mathrm{CRF02} \mathrm{AG}(1.7), \mathrm{AD}(1.7), \mathrm{AG} \\
\text { (1.7) }\end{array}$ \\
\hline Libya & 68 & CRF02_AG (100) \\
\hline Morocco & 2 & $B(100)$ \\
\hline Saudi Arabia & 169 & $\begin{array}{l}\text { C (36.7), G (24.3), B (18.3), D (4.7), } \\
\text { CRF43_02G (4.7), A (4.1), CRF02_AG } \\
\text { (3.6), CRF25_cpx (2.4), other (1.2) }\end{array}$ \\
\hline Somalia & 24 & C (87.5), AC (12.5) \\
\hline Sudan & 40 & $\mathrm{D}(55), \mathrm{C}(30), \mathrm{A}(7.5), \mathrm{B}(5), \mathrm{U}(2.5)$ \\
\hline Tunisia & 24 & B (95.8), CRF02_AG (4.2) \\
\hline Yemen & 25 & B (48), C (28), D (16), A (4), U (4) \\
\hline
\end{tabular}

${ }^{*}$ Frequency in the HIV database and not necessarily in the respective population. MENA, Middle East and North Africa.

genetic variants closely related to those found over a wide geographical range, including Europe, North America, Asia and Africa. $^{30}$

\section{Settings with dominance of specific subtypes}

In a few countries, a trend of a dominant HIV-1 subtype was observed and linked to HIV transmission chains within specific high-risk groups such as among IDU in Afghanistan, Iran and Pakistan (table 1). In Iran, all viral sequences identified among IDU in two different cities formed a single cluster within subtype A and were found to be related, although not necessarily directly, to Ugandan/Kenyan isolates. ${ }^{12} 1332$ Interestingly, there was a lack of strong phylogenetic correlation between the sequences from Iran and those from other Middle Eastern countries, apart from Afghanistan, where subtype A is common. ${ }^{13} 32$ The confined variability in the genes suggests the relatively recent nature of the epidemic in Iran. ${ }^{12} 132$ Similarly, subtype A was the only clade observed among a group of IDU in Karachi, Pakistan, where the isolated viruses formed a tight monophyletic group indicating a founder effect. ${ }^{23} 33$ In more recent studies among IDU in Iran, ${ }^{14-16}$ CRF35_AD was identified; this subtype had also recently been identified among a small group of IDU in Kabul, Afghanistan. ${ }^{8}$

Clustering within other subtypes was also observed in some MENA countries. In Djibouti, 73\% of infections in 2002 were of subtype C, which had previously been described among FSW in the horn of Africa. ${ }^{10}$ Subtype $\mathrm{C}$ was found in $48 \%$ of French military personnel infected with HIV who served in Djibouti. ${ }^{11}$ Similarly, subtype C accounted for $87.5 \%$ of HIV-1 cases in Somalia (table 2). Sudan was the only country in MENA where subtype $\mathrm{D}$ was observed at a high frequency of $50 \%{ }^{27}$

Subtype B was found to be the prevalent subtype in a number of MENA countries. In Algeria subtype B was found to be predominant, particularly in the north of the country, accounting for $56 \%$ of samples studied. ${ }^{9}$ There is, however, a high diversity of recombinant subtypes in the southern part of
Algeria, bordering with sub-Saharan Africa. ${ }^{9}$ Subtype B also accounted for $98 \%$ of the cases among Caucasian Israeli and Palestinian isolates. ${ }^{17}$ In Tunisia, $95 \%$ of HIV-1 infections were found to be of subtype B and had probable origins in western Europe $^{28}$ Subtype B was historically also found to be the dominant strain in Morocco, accounting for $93.5 \%$ of infections in the 1990s and reflecting transmission pathways of western European origins. ${ }^{20}$ However, more recent data indicate that although subtype B remains the predominant strain, there is an increasing diversity of HIV-1 variants, with over $25 \%$ of the infections being of non-B subtypes, including several CRF. ${ }^{22}$ In one study from Morocco, $21.4 \%$ of the infections were of subtype A and clustered with strains of Kenyan origin. ${ }^{21}$

\section{DISCUSSION \\ General overview}

It is not straightforward to draw firm conclusions from available studies, especially as many are based on an analysis of a small number of isolates, which in some settings may be reflecting transmission patterns from the past rather than current ongoing transmission (table 1). However, the gathered molecular data on HIV-1 subtype distribution in the region, substantiated by other epidemiological evidence on disease distribution, HIV dynamics among high-risk populations and risk networks in respective countries (G Mumtaz, 2010, unpublished data), ${ }^{56}$ point towards two major patterns of HIV acquisition and transmission in MENA countries: one of random exogenous exposures related to travel history, which is present all over MENA (figure 1A and $1 \mathrm{~B}$ ), and another of local epidemic-type clustering related to concentrated HIV epidemics in specific high-risk groups in a number of MENA countries (figure 1B).

\section{Patterns of exogenous HIV exposures}

In a number of MENA countries, as was observed in Lebanon, Saudi Arabia and Yemen, sequence analyses indicated multiple distinct subtypes in the isolated strains, suggesting multiple travel-related introductions rather than the endemic transmission of genetically diverse HIV-1 subtypes (figure 1A). In some other settings such as in northern Algeria, Palestine and Tunisia, although one subtype is predominant, the intrasubtype diversity suggests that HIV transmission appears to be strongly affected by multiple introductions, but from confined geographical sites. In both kinds of settings, HIV dynamics, based strictly on molecular evidence, do not appear to be dominated by a specific considerable epidemic in one or multiple high-risk groups (figure $1 \mathrm{~A}$ ).

The high diversity in subtypes and/or the high genetic divergence among any specific subtype suggest that HIV infections in these countries are driven by travel-related exogenous exposures among nationals or HIV acquisition by their sexual partners upon their return. ${ }^{5}$ Data from case notification reports confirm this postulated trend. ${ }^{34}$ For example in Lebanon, $45.4 \%$ of notified cases up to 2004 were linked to travel abroad, ${ }^{35}$ as were half of reported AIDS cases in Yemen. ${ }^{34}$ In these settings, local spread is usually limited to immediate sexual partners and does not lead to an epidemic. ${ }^{36}$ In one study in Saudi Arabia, a strong phylogenetic relationship between HIV-1 strains was observed among several husband-wife pairs, indicating heterosexual transmission between spouses. ${ }^{25}$ Indeed, $97 \%$ of HIVinfected women in Saudi Arabia who acquired HIV sexually acquired the infection from their husbands. ${ }^{37}$ This trend of travel-associated infections has also been observed in other countries outside of MENA, such as in Cuba where the epidemic is characterised by a high diversity of subtypes mainly due to the 

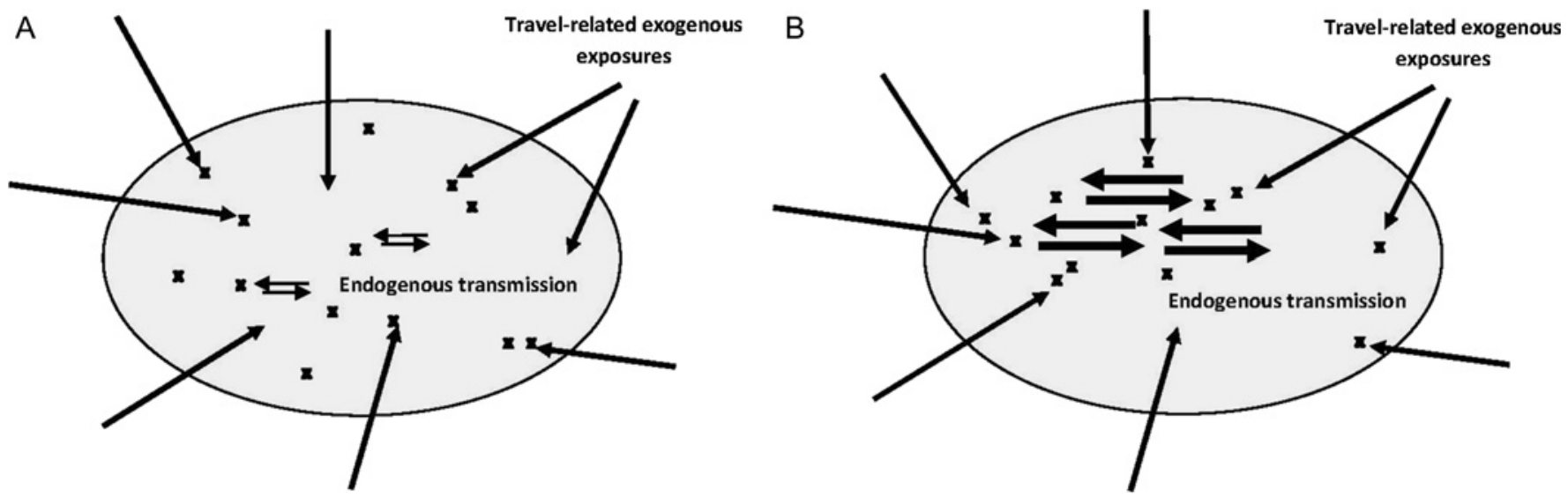

Figure 1 Schematic representation of countries with dominance of travel-related exogenous HIV exposures (A), and dominance of endogenous epidemic-type HIV transmissions in addition to the contribution of travel-related exogenous HIV exposures (B). The rate and number of exogenous exposures varies between Middle East and North Africa countries.

return of Cuban military troops from Angola, the large numbers of Cuban aid workers serving in sub-Saharan countries, and the recent development of tourist resorts in the country. ${ }^{36} 38$

The high population mobility in MENA including migration, tourism, studying and working abroad, and military deployment among others explain in large part the observed pattern of exogenous exposures and subsequent subtype diversity, ${ }^{6}$ as well as explaining the observed Africanisation of subtypes in some settings. $^{6} \quad 92225$ Southern Algeria and Morocco are both important migration hubs for migrants from sub-Saharan African countries towards Europe and this is reflected in the high diversity of non-B subtypes and CRF in these regions. ${ }^{9} 22$ In Turkey, non-B subtype infections are also thought to be mainly caused by immigrants or residents from Africa, the Balkans and the Middle East. ${ }^{29}$ The high prevalence of subtype C in Saudi Arabia is expected in view of the country's ties with neighbouring Horn of Africa countries. ${ }^{25}$ The very high diversity of subtypes observed in Lebanon reflects the frequent population movements and return of expatriates such as from West Africa. ${ }^{18}$ The high level of emigration to western Europe explains the predominance of subtype B in Tunisia, which is thought to have been initially introduced mainly by young male IDU or individuals with haemophilia infected in Europe. ${ }^{28}$

\section{Patterns of local epidemic-type clustering}

On the other hand, the dominance of specific subtypes with limited sequence variability in Afghanistan, Djibouti, Iran, Libya, Pakistan and Somalia in addition possibly to one subcluster in Saudi Arabia, suggests that HIV acquisitions in some countries are occurring in transmission chains that have been propagating locally for at least a number of years. This suggests active high-risk networks where HIV is being transmitted via typical founder effect, which translates into local epidemic-type clustering in one or multiple risk groups (figure 1B). ${ }^{8} 101213233233$ This trend was documented in Russia, for example, where injecting drug use represented the main mode of HIV transmission for the longest time and where subtype A was the predominant clade driving the epidemic among IDU. ${ }^{39}$

This conjecture is supported in Iran and Pakistan as examples, by robust evidence for concentrated epidemics among IDU. ${ }^{5}$ By the end of 2007, IDU accounted for at least $67 \%$ of HIV notified cases in Iran. ${ }^{40}$ In Pakistan, the contribution of IDU to the total notified cases has been rising, and IDU accounted for the largest proportion of HIV infections in $2008 .^{41}$ The similarities between
HIV-1 subtypes in Afghanistan, Iran and apparently Pakistan suggest that strains have been circulating between these countries, probably through the return of Afghani refugees, and may indicate that social networks of drug producers, refiners, distributors and IDU transcend national borders. ${ }^{8}$

The local epidemic-type clustering is also supported by epidemiological evidence from Djibouti and from some parts of Somalia, where there is evidence of concentrated HIV epidemics in commercial heterosexual sex networks, ${ }^{5}$ with rates of HIV up to $70 \%$ being reported among street-based FSW in Djibouti. ${ }^{42} 43$ Clustering within subtype C in HIV-1 infections in Djibouti and Somalia is in accordance with previous studies in the Horn of Africa, which have identified subtype $\mathrm{C}$ to be the dominant clade, accounting for up to $98.5 \%$ of infections among FSW in Ethiopia. ${ }^{44}{ }^{45}$ Similarly, in Sudan the epidemic appears to be dominated by East African viruses also circulating in neighbouring countries. ${ }^{27}$ Libya is yet another example where clustering of subtypes was observed, but in the context of a long-standing nosocomial transmission scenario. ${ }^{19}$

\section{Transmission links between epidemics}

One of the insightful applications of HIV molecular research is that it can indicate whether there is a link between two epidemics occurring simultaneously in two different risk groups in one country. This was reported in many regions of the world such as in Thailand, where in the first decade of the epidemic, HIV-1 subtypes were segregated by risk group, with IDU being infected with subtype $B$ and patients infected through the heterosexual route being infected with CRF01_AE. ${ }^{46} 47$

Similarly in Iran, molecular epidemiology investigations indicated that the HIV epidemic among IDU is independent of the epidemic among individuals with haemophilia as seen by the different subtype clustering in each transmission network: IDU were found to be exclusively infected with subtype A, which has been possibly introduced to Iranian IDU by a pilgrim visiting Mashhad, Iran's holiest city, ${ }^{32}$ whereas individuals with haemophilia were exclusively infected by subtype B. ${ }^{12}$ In Israel/ Palestine, whereas subtype $\mathrm{B}$ was the predominant clade, subtype $\mathrm{C}$ was exclusively observed among the Ethiopian Israeli population, indicating two distinct HIV transmission pathways in the population that possibly reflect the geographical site of virus acquisition. ${ }^{17}$

Recent phylogenetic analyses have also linked epidemics in MENA such as the emerging epidemic among MSM in Pakistan 


\section{Key messages}

- HIV molecular epidemiology investigation is a powerful tool for tracking transmission patterns and the spread and evolution of the epidemic in a population.

- This is the first review of all available data on HIV-1 subtype distribution in MENA and implications in terms of transmission patterns.

- Molecular evidence shows two main patterns: the dominance of exogenous exposures in several countries and the dominance of local epidemic-type clustering among high-risk groups in others.

- The virus subtype distribution in MENA reflects the transmission pathways of virus introduction into this region from the other parts of the world.

and that which emerged among IDU earlier in this decade in this country (S. Ali, personal communication, 2010).

\section{Recommendations for future research and interventions}

With the weak HIV surveillance systems in this region, it is useful to expand HIV molecular research, especially as some of the available studies have been conducted a decade ago. Overall in MENA, only a very small number of HIV cases have ever been subtyped. Despite the high cost of molecular analyses, identifying the subtypes and studying the phylogenetic clustering of at least small subsamples of notified HIV cases and HIV-seropositive cases in surveyed populations is pertinent. It can pinpoint the most affected risk groups and the prevalent modes of transmission in the population, and help establish some reliable information on the subtype distributions. However, the priority in MENA countries that are still developing their HIV surveillance infrastructure would be to invest in integrated biobehavioural surveillance using state of the art sampling methodologies for hard-to-reach populations. This kind of surveillance is essential in order to facilitate the prioritisation of HIV response to where needs are and to avert the possibility of HIV efforts being dissipated on non-consequential programmes.

The molecular evidence gathered in this review, supported by other epidemiological evidence (G Mumtaz, 2010, unpublished data), ${ }^{5}{ }^{6}$ indicates the absence of a sustainable general population HIV epidemic in MENA, with the exception possibly of southern Sudan. As such, any current HIV prevention efforts focused on the general population are misplaced. Whereas it is challenging to identify, prevent or even control travel-related exogenous exposures, the number of infections arising from these random introductions remains relatively small. Instead, surveillance and prevention efforts should be prioritised for highrisk groups including IDU, MSM and FSW, in whom low intensity and concentrated epidemics are emerging or are already established. This would prevent further HIV transmission or the emergence of localised and hidden HIV epidemics among these population groups.

Funding The MENA HIV/AIDS Epidemiology Synthesis Project was funded through a joint partnership of the World Bank, the MENA Regional Support Team of the Joint United Nations Programme on HIV/AIDS (UNAIDS) and the Eastern Mediterranean Regional Office (EMRO) of the WHO. LJA and GM are also grateful for the Oatar National Research Fund for supporting this work (NPRP 08-068-3-024).

\section{Competing interests None.}

Contributors LJA conceived and led the design and conduct of this study; co-led the literature reviews and collection, analysis and interpretation of the data; and contributed to the drafting of the manuscript. GM led the drafting of the manuscript and co-led the literature reviews and collection, analysis and interpretation of the data. $\mathrm{NH}$ contributed to the literature reviews and assisted in the general conduct of the research. FAA, IS, GR and DW contributed to the interpretation of the results, contributed to the drafting of the manuscript, and provided data at the regional and country level. All authors were involved in the finalisation of the manuscript and approved the final version.

Provenance and peer review Not commissioned; externally peer reviewed.

\section{REFERENCES}

1. Woodman Z, Williamson C. HIV molecular epidemiology: transmission and adaptation to human populations. Curr Opin HIV AIDS 2009;4:247-52.

2. Buonaguro L, Tornesello ML, Buonaguro FM. Human immunodeficiency virus type 1 subtype distribution in the worldwide epidemic: pathogenetic and therapeutic implications. J Virol 2007;81:10209-19.

3. Papathanasopoulos MA, Hunt GM, Tiemessen CT. Evolution and diversity of HIV-1 in Africa - a review. Virus Genes 2003;26:151-63.

4. Tatt ID, Barlow KL, Nicoll A, et al. The public health significance of HIV-1 subtypes. AIDS 2001;15(Suppl 5):S59-71.

5. Abu-Raddad LJ, Hilmi N, Mumtaz G, et al. Epidemiology of HIV infection in the Middle East and North Africa. AIDS 2010;24(Suppl 2):S5-23.

6. Abu-Raddad L, Akala FA, Semini l, et al. Characterizing the HIVIAIDS epidemic in the Middle East and North Africa: time for strategic action. Middle East and North Africa HIVIAIDS Epidemiology Synthesis Project. Washington DC, USA: World Bank/UNAIDS/WHO Publication, 2010.

7. Los Alamos HIV database, National Institute of Allergy and Infectious Diseases (NIAID). http://www.hiv.lanl.gov/content/index (accessed Feb 2010).

8. Sanders-Buell E, Saad MD, Abed AM, et al. A nascent HIV type 1 epidemic among injecting drug users in Kabul, Afghanistan is dominated by complex $A D$ recombinant strain, CRF35 AD. AIDS Res Hum Retroviruses 2007;23:834-9.

9. Bouzeghoub S, Jauvin V, Recordon-Pinson P, et al. High diversity of HIV type 1 in Algeria. AIDS Res Hum Retroviruses 2006;22:367-72.

10. Maslin J, Rogier C, Berger F, et al. Epidemiology and genetic characterization of HIV 1 isolates in the general population of Djibouti (Horn of Africa). J Acquir Immune Defic Syndr 2005;39:129-32.

11. Lasky M, Perret JL, Peeters $M$, et al. Presence of multiple non-B subtypes and divergent subtype $B$ strains of HIV-1 in individuals infected after overseas deployment. AIDS 1997;11:43-51.

12. Sarrami-Forooshani R, Das SR, Sabahi F, et al. Molecular analysis and phylogenetic characterization of HIV in Iran. J Med Virol 2006;78:853-63.

13. Naderi HR, Tagliamonte M, Tornesello ML, et al. Molecular and phylogenetic analysis of HIV-1 variants circulating among injecting drug users in Mashhad, Iran. Infect Agent Cancer 2006;1:4.

14. Soheilli ZS, Ataiee Z, Tootian S, et al. Presence of HIV-1 CRF35 AD in Iran. AIDS Res Hum Retroviruses 2009;25:123-4.

15. Mousavi SM, Hamkar R, Gouya MM, et al. Surveillance of HIV drug resistance transmission in Iran: experience gained from a pilot study. Arch Virol 2010;155:329-34.

16. Hamkar R, Mohraz M, Lorestani S, et al. Assessing subtype and drug-resistance associated mutations among antiretroviral-treated HIV-infected patients. AIDS 2010;24(Suppl 2):S85-91.

17. Gehring S, Maayan S, Ruppach $\mathrm{H}$, et al. Molecular epidemiology of HIV in Israel. J Acquir Immune Defic Syndr Hum Retrovirol 1997;15:296-303.

18. Pieniazek D, Baggs J, Hu DJ, et al. Introduction of HIV-2 and multiple HIV-1 subtypes to Lebanon. Emerg Infect Dis 1998;4:649-56.

19. de Oliveira T, Pybus OG, Rambaut A, et al. Molecular epidemiology: HIV-1 and HCV sequences from Libyan outbreak. Nature 2006;444:836-7.

20. Elharti E, Elaouad R, Amzazi S, et al. HIV-1 diversity in Morocco. AIDS 1997:11:1781-3.

21. Abid M, Luo CC, Sekkat $S$, et al. Characterization of the V3 region of HIV type 1 isolates from Morocco. AIDS Res Hum Retroviruses 1998;14:1387-9.

22. Bakhouch K, Oulad-Lahcen A, Bensghir R, et al. The prevalence of resistanceassociated mutations to protease and reverse transcriptase inhibitors in treatment-naive (HIV1)-infected individuals in Casablanca, Morocco. J Infect Dev Ctries 2009:3:380-91.

23. Khan S, Rai MA, Khanani MR, et al. HIV-1 subtype A infection in a community of intravenous drug users in Pakistan. BMC Infect Dis 2006;6:164.

24. Yamaguchi J, Badreddine S, Swanson P, et al. Identification of new CRF43 O2G and CRF25_cpx in Saudi Arabia based on full genome sequence analysis of six HIV type 1 isolates. AIDS Res Hum Retroviruses 2008;24:1327-35.

25. Badreddine S, Smith K, van Zyl H, et al. Identification and characterization of HIV type 1 subtypes present in the Kingdom of Saudi Arabia: high level of genetic diversity found. AIDS Res Hum Retroviruses 2007;23:667-74.

26. Alzahrani AJ. Analysis of HIV subtypes and the phylogenetic tree in HIV-positive samples from Saudi Arabia. Saudi Med J 2008;29:1394-6.

27. Hierholzer M, Graham RR, El Khidir I, et al. HIV type 1 strains from East and West Africa are intermixed in Sudan. AIDS Res Hum Retroviruses 2002;18:1163-6.

28. Ben Halima M, Pasquier C, Slim A, et al. First molecular characterization of HIV-1 Tunisian strains. J Acquir Immune Defic Syndr 2001;28:94-6.

29. Yilmaz G, Midilli K, Turkoglu $S$, et al. Genetic subtypes of human immunodeficiency virus type 1 (HIV-1) in Istanbul, Turkey. Int J Infect Dis 2006;10:286-90.

30. Saad MD, Al-Jaufy A, Grahan RR, et al. HIV type 1 strains common in Europe, Africa, and Asia cocirculate in Yemen. AIDS Res Hum Retroviruses 2005;21:644-8. 
31. Thomson MM, Najera R. Molecular epidemiology of HIV-1 variants in the global AIDS pandemic: an update. AIDS Rev 2005;7:210-24.

32. Tagliamonte $\mathbf{M}$, Naderi HR, Tornesello ML, et al. HIV type 1 subtype A epidemic in injecting drug user (IDU) communities in Iran. AIDS Res Hum Retroviruses 2007;23:1569-74.

33. Rai MA, Nerurkar VR, Khoja S, et al. Evidence for a "Founder Effect" among HIV-infected injection drug users (IDUs) in Pakistan. BMC Infect Dis 2010;10:7.

34. WHO, UNICEF, UNAIDS, Yemen. Epidemiological facts sheets on HIVIAIDS and sexually transmitted infections. Geneva, Switzerland: UNAIDS, 2006.

35. Karam W, Aftimos G, Jurjus A, et al. Prevalence of sexually transmitted infections in Lebanese women as revealed by pap smear cytology: a cross sectional study from 2002-2006. Cairo, Egypt, WHO/EMRO 2007.

36. Perrin L, Kaiser L, Yerly S. Travel and the spread of HIV-1 genetic variants. Lancet Infect Dis 2003;3:22-7.

37. Alrajhi AA, Halim MA, Al-Abdely HM. Mode of transmission of HIV-1 in Saudi Arabia. AIDS 2004;18:1478-80.

38. Cuevas MT, Ruibal I, Villahermosa ML, et al. High HIV-1 genetic diversity in Cuba. AIDS 2002;16:1643-53.

39. Thomson MM, Vinogradova A, Delgado E, et al. Molecular epidemiology of HIV-1 in St Petersburg, Russia: predominance of subtype A, former Soviet Union variant, and identification of intrasubtype subclusters. J Acquir Immune Defic Syndr 2009;51:332-9.

40. Iran Ministry of Health. AIDS/HIV surveillance report, fourth quarter. Teheran: Ministry of Health, 2007.

41. Pakistan Ministry of Health. AIDS/HIV surveillance report, third quarter Islamabad, Pakistan, 2008

42. Marcelin AG, Grandadam M, Flandre $\mathrm{P}$, et al. Comparative study of heterosexual transmission of HIV-1, HSV-2 and KSHV in Diibouti. 8th Retrovir Oppor Infect labstract no. 585). Foundation for Retrovirology and Human Health. Chicago, Illinois, USA. 4-8 February 2001

43. Marcelin AG, Grandadam M, Flandre P, et al. Kaposi's sarcoma herpesvirus and HIV-1 seroprevalences in prostitutes in Dijbouti. J Med Virol 2002:68:164-7.

44. Hussein M, Abebe A, Pollakis G, et al. HIV-1 subtype C in commerical sex workers in Addis Ababa, Ethiopia. J Acquir Immune Defic Syndr 2000;23:120-7.

45. Zewde A, Bahiru S, Sanders E, et al. HIV-1 seroprevalence and subtypes in police recruits from Afar regional state, Ethiopia. Ethiop Med J 2002;40(Suppl 1):1-10.

46. Ou CY, Takebe Y, Weniger BG, et al. Independent introduction of two major HIV-1 genotypes into distinct high-risk populations in Thailand. Lancet 1993;341:1171-4.

47. Arroyo MA, Phanuphak N, Krasaesub S, et al. HIV type 1 molecular epidemiology among high-risk clients attending the Thai Red Cross Anonymous Clinic in Bangkok, Thailand. AIDS Res Hum Retroviruses 2010;26:5-12. 